\title{
Plasmacytosis and nephrotic syndrome revealing Sjögren's syndrome
}

\author{
Céline La $(D)$, Muhammad Soyfoo (1) \\ Department of Rheumatology, Hôpital Erasme, Brussels, Belgium
}

Sjögren's syndrome is an autoimmune disease characterized by the lymphocytic infiltration of exocrine glands resulting in dry eyes and mouth but can also cause multi-organ damage. ${ }^{1}$ Renal involvement is rare during primary Sjögren's syndrome, occurring in 3 to $5 \%$ of patients, and even rarely resulting in end-stage renal disease $(5 \%) .^{2-5}$

We herein report the case of a 43-year-old female patient admitted to our hospital with sudden onset dyspnea, abdominal pain, diffuse edema, weight gain of $7 \mathrm{~kg}$, dyspepsia and polyarthralgia. The patient had been recently diagnosed with systemic erythematosus lupus based on polyarthralgia, normocytic anemia and positivity of antinuclear antibody and anti-Sjögren's syndrome type A (anti-SSA) antibody. The laboratory investigations showed an acute kidney injury with nephrotic syndrome, anti-SSA and antiSjögren's syndrome type B (anti-SSB) antibody positivity, low complement component 4 levels and hypergammaglobulinemia. Cryoglobulinemia was excluded. A percutaneous kidney biopsy was performed the day after her admission. The patient was rapidly treated with high-doses of intravenous corticosteroids, but her clinical condition and renal failure worsened with an estimated glomerular filtration rate of $19 \mathrm{~mL} / \mathrm{min}$. The kidney biopsy revealed acute tubulointerstitial nephritis with massive lymphoplasmacytic infiltrate positive for anti-cluster of differentiation 20 antibody and no expression of anti-immunoglobulin G4 antibody (Figure 1a, b). Because of the important lymphocytic infiltrate, a bone marrow biopsy was performed to exclude lymphoma and showed a rich marrow cellularity with polyclonal plasmacytosis (Figure 1c, d). Positron emission tomography (PET)-fluorodeoxyglucose (FDG) scan did not demonstrate any enlarged lymph nodes or hepatosplenomegaly in favor of lymphoma or Castleman disease. Schirmer's test came back positive and a minor salivary gland biopsy depicted lymphocytic sialadenitis with a Chisholm and Mason score of 4 (Figure 1e, f). Electromyography results showed inflammatory polyradiculoneuritis in $\mathrm{C} 6$ and C7. A gastric emptying scan was consistent with gastroparesis. Moreover, immunohistochemistry results of the kidney biopsy also came back negative for human herpesvirus 8 (HHV8) antibody. The absence of the typical histological signs, as well as HHV8 negativity and normal PET-FDG ruled out Castleman disease. Our patient presented primary Sjögren's syndrome with complex systemic manifestations, including renal, hematological (lymphoproliferative syndrome), neurological (polyradiculoneuritis and gastroparesis) and articular disease. The patient was treated with

Received: July 27, 2020 Accepted: September 16, 2020 Published online: December 10, 2020

Correspondence: Céline La, MD. Department of Rheumatology, Hôpital Erasme, 1070 Brussels, Belgium. Tel: +32 25553650 e-mail: celine.la@erasme.ulb.ac.be

\section{Citation:}

La C, Soyfoo M. Plasmacytosis and nephrotic syndrome revealing Sjögren's syndrome. Arch Rheumatol 2021;36(2):311-313. 

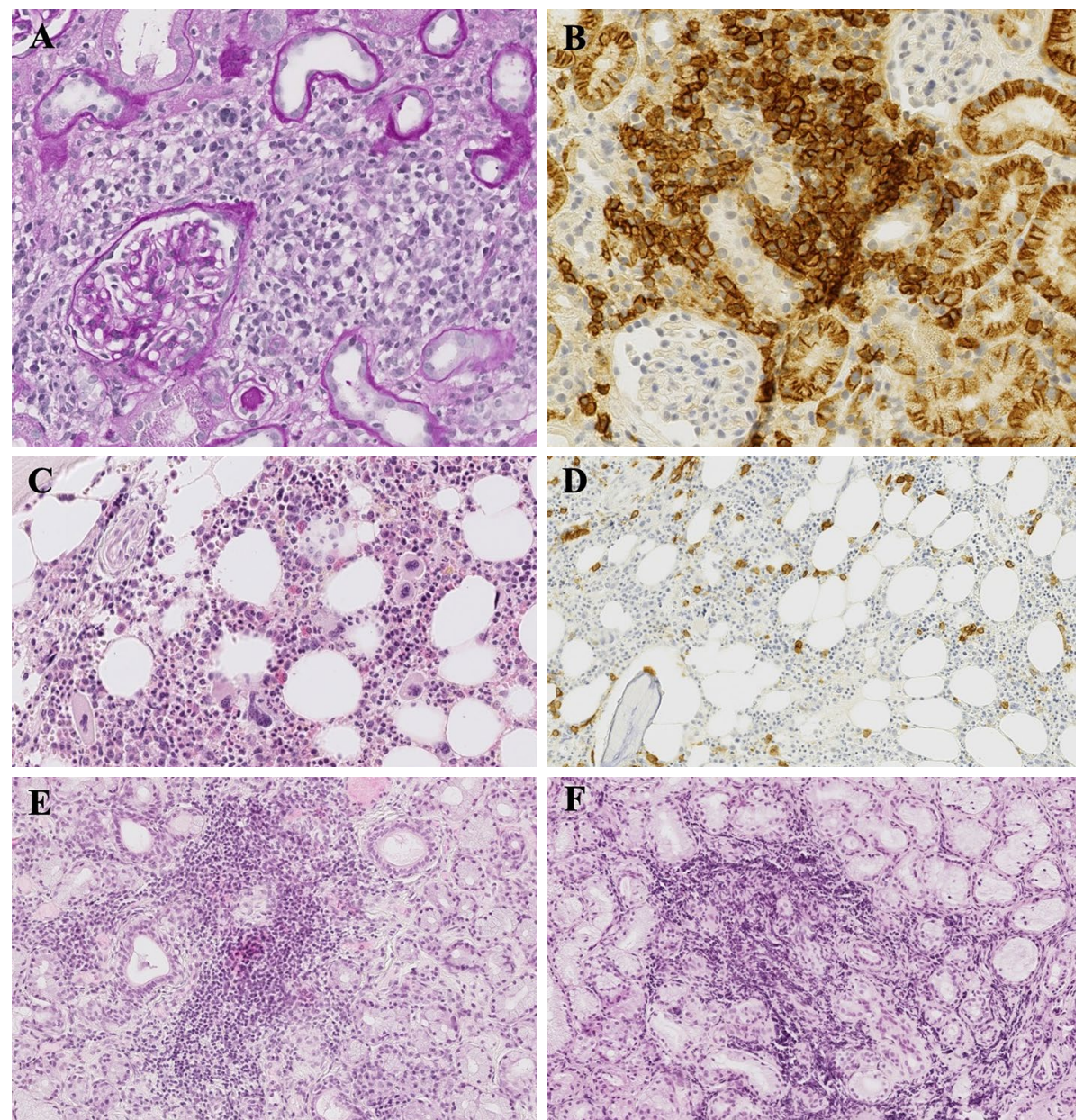

Figure 1. (a) Histopathologic images of kidney biopsy show a massive lymphoplasmacytic infiltrate disrupting renal parenchyma and renal tubules but sparing glomeruli. (b) Immunostaining of CD 138 in kidney biopsy confirms plasmacytic nature of tubulointerstitial infiltrate. (c) Histopathologic pictures of bone marrow biopsy show a rich cellularity, with all three lineages represented. (d) Immunostaining of CD138 in bone marrow biopsy indicates a mild plasmacytosis without monoclonality. (e, f) Salivary gland biopsy showed a lymphocytic sialadenitis with a Chisholm and Mason score of 4.

CD: Cluster of differentiation.

rituximab and oral steroids with good clinical and biological improvement. Cyclophosphamide and steroids were started a year later for a renal flare but suspended because of a major hematological toxicity and the patient had to undergo chronic hemodialysis. Finally, she received a renal transplant from a non-heart-beating-donor five years after initial diagnosis and is currently treated with tacrolimus and mycophenolate. A written informed consent was obtained from the patient.

In conclusion, renal involvement is rare in primary Sjögren's syndrome. Tubulointerstitial nephritis represents the largest majority of cases, while glomerular lesions are rather associated with cryoglobulinemia. ${ }^{6,7}$ The prognosis is usually favorable, but the patients should be carefully screened if renal involvement is suspected, because of the risk of developing chronic kidney disease. Furthermore, a renal biopsy should be discussed to exclude other causes of renal insufficiency and to guide the therapeutic decision.

\section{Declaration of conflicting interests}

The authors declared no conflicts of interest with respect to the authorship and/or publication of this article. 


\section{Funding}

The authors received no financial support for the research and/or authorship of this article.

\section{REFERENCES}

1. Brito-Zerón P, Baldini C, Bootsma H, Bowman SJ, Jonsson R, Mariette X, et al. Sjögren syndrome. Nat Rev Dis Primers 2016;2:16047.

2. Goules AV, Tatouli IP, Moutsopoulos HM, Tzioufas AG. Clinically significant renal involvement in primary Sjögren's syndrome: clinical presentation and outcome. Arthritis Rheum 2013;65:2945-53.

3. Ramos-Casals M, Brito-Zerón P, Solans R, Camps MT, Casanovas A, Sopeña B, et al. Systemic involvement in primary Sjogren's syndrome evaluated by the EULAR-SS disease activity index: analysis of 921
Spanish patients (GEAS-SS Registry). Rheumatology (Oxford) 2014;53:321-31.

4. Baldini C, Pepe P, Quartuccio L, Priori R, Bartoloni $\mathrm{E}$, Alunno A, et al. Primary Sjogren's syndrome as a multi-organ disease: impact of the serological profile on the clinical presentation of the disease in a large cohort of Italian patients. Rheumatology (Oxford) 2014;53:839-44.

5. François $\mathrm{H}$, Mariette X. Renal involvement in primary Sjögren syndrome. Nat Rev Nephrol 2016;12:82-93.

6. Jasiek M, Karras A, Le Guern V, Krastinova E, Mesbah R, Faguer S, et al. A multicentre study of 95 biopsy-proven cases of renal disease in primary Sjögren's syndrome. Rheumatology (Oxford) 2017;56:362-70.

7. Jain A, Srinivas BH, Emmanuel D, Jain VK, Parameshwaran S, Negi VS. Renal involvement in primary Sjogren's syndrome: a prospective cohort study. Rheumatol Int 2018;38:2251-62. 